\title{
Enhancement of micro-channels within the human mastoid bone based on local structure tensor analysis
}

\author{
Olivier Cros, Anders Eklund, Michael Gaihede and Hans Knutsson \\ Conference Publication
}

\section{Tweet}

N.B.: When citing this work, cite the original article.

Original Publication:

Olivier Cros, Anders Eklund, Michael Gaihede and Hans Knutsson, Enhancement of microchannels within the human mastoid bone based on local structure tensor analysis, Image Proceessing Theory, Tools and Apllications, 2016. (), pp..

http://dx.doi.org/10.1109/IPTA.2016.7821019

Copyright: www.ieee.org

Postprint available at: Linköping University Electronic Press

http://urn.kb.se/resolve?urn=urn:nbn:se:liu:diva-134434

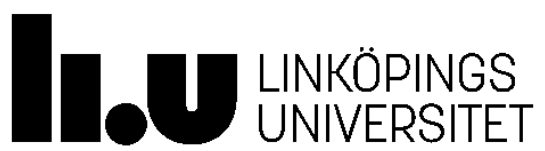




\title{
Enhancement \\ of micro-channels within the human mastoid bone based on local structure tensor analysis
}

\author{
Olivier Cros ${ }^{124}$, Anders Eklund ${ }^{123}$, Michael Gaihede ${ }^{45}$ and Hans Knutsson ${ }^{12}$ \\ ${ }^{1}$ Department of Biomedical Engineering, Linköping University, Sweden \\ e-mail: olivier.cros@liu.se, \\ 2 Center for Medical Image Science and Visualization (CMIV), Linköping University, Sweden \\ ${ }^{3}$ Department of Computer and Information Science, Linköping University, Sweden \\ ${ }^{4}$ Department of Otolaryngology, Head \& Neck Surgery, Aalborg University Hospital, Denmark \\ ${ }^{5}$ Department of Clinical Medicine, Aalborg University, Denmark
}

\begin{abstract}
Numerous micro-channels have recently been discovered in the human temporal bone by $x$-ray micro-CTscanning. After a preliminary study suggesting that these microchannels form a separate blood supply for the mucosa of the mastoid air cells, a structural analysis of the micro-channels using a local structure tensor was carried out. Despite the highresolution of the micro-CT scan, presence of noise within the air cells along with missing information in some micro-channels suggested the need of image enhancement. This paper proposes an adaptive enhancement of the micro-channels based on a local structure analysis while minimizing the impact of noise on the overall data. Comparison with an anisotropic diffusion PDE based scheme was also performed.
\end{abstract}

Keywords-Structure tensor analysis, Image enhancement, Adaptive filtering, Human temporal bone, Mastoid bone, Microchannels.

\section{INTRODUCTION}

In the middle ear, pressure is passively regulated through gas exchange via a respiratory mucosa lining the bone. The tympanum, part of the middle ear and in direct communication with the mastoid air cell system (MACS), is known to be the main structure involved in this regulatory process. However, more recent studies have indicated that the MACS also plays an important role in pressure regulation [1], [2]. To allow an efficient gas exchange, such as in the lungs, a large surface area of respiratory mucosa is needed in relation to the volume of air contained within the air cavities. To investigate how large the surface area and the volume of the MACS are, eight human temporal bone specimens were scanned using X-ray micro-CT scanning [1]. Part of a right human temporal bone is visualized using volume rendering in Fig. 2. Among other landmarks, so as to further help the reader, the MACS is located to the left, the tympanum is located in the lower right, the cochlea and semi-circular canals are visible to the top right, while the ear canal appears in the lower part of Fig. 2. Further investigation of the micro-CT scan led to the discovery of micro-channels within the bony part of the mastoid between the MACS, visible as small dark features, notably at the vicinity of the ear canal [1], see Fig. 2. A possible role of these microchannels is a secondary blood supply to the mucosa lining

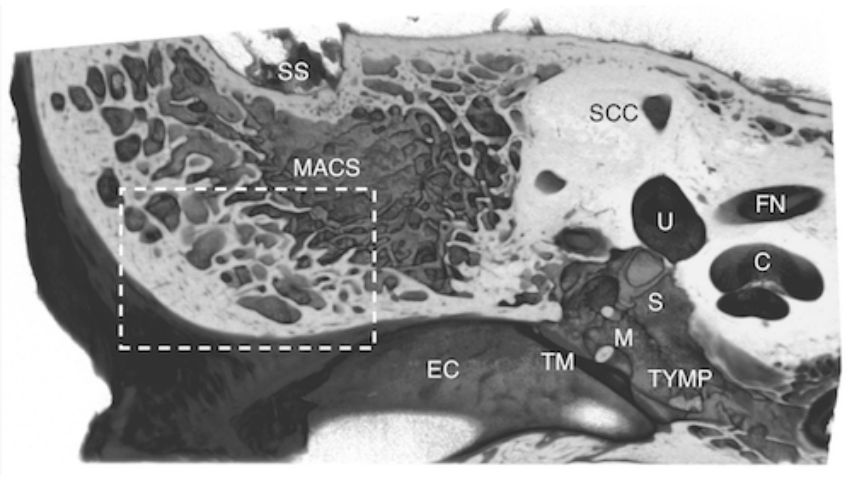

Fig. 1. Volume rendering of an axial view of a right human temporal bone specimen with well-known landmarks: cochlea (C), ear canal (EC), facial nerve (FN), malleus (M), mastoid air cell system (MACS), utricle (U), semi-circular canals (SCC), sigmoid sinus (SS), staple (S), tympanic membrane (TM), and the tympanum (TYMP). The dotted rectangle indicates the approximate region used during the study.

the MACS, to allow a more efficient pressure regulation, see Fig. 2 where the micro-channels are observed within the white ellipsoid at the vicinity of the ear canal (EC). In order to gain further knowledge about these micro-channels, a geometrical analysis has been carried out using a micro-CT scan of a single human temporal bone specimen [3]. The extraction of tubular and planar structures and combinations thereof, using local structure tensor analysis ([5]), revealed microchannels of different size and shape. While larger microchannels were well described, micro-channels of smaller size appeared disrupted by the presence of noise in the data, leading to disconnected structures in the resulting structure tensor. Furthermore, presence of noise in the data prevents a clear visualisation of the micro-channels path within the bone and their linking to adjacent air cells. This study therefore proposes an extension of the local structure tensor analysis ([3]), by enhancing the micro-CT data, while reducing the noise level using adaptive filtering [6]. 


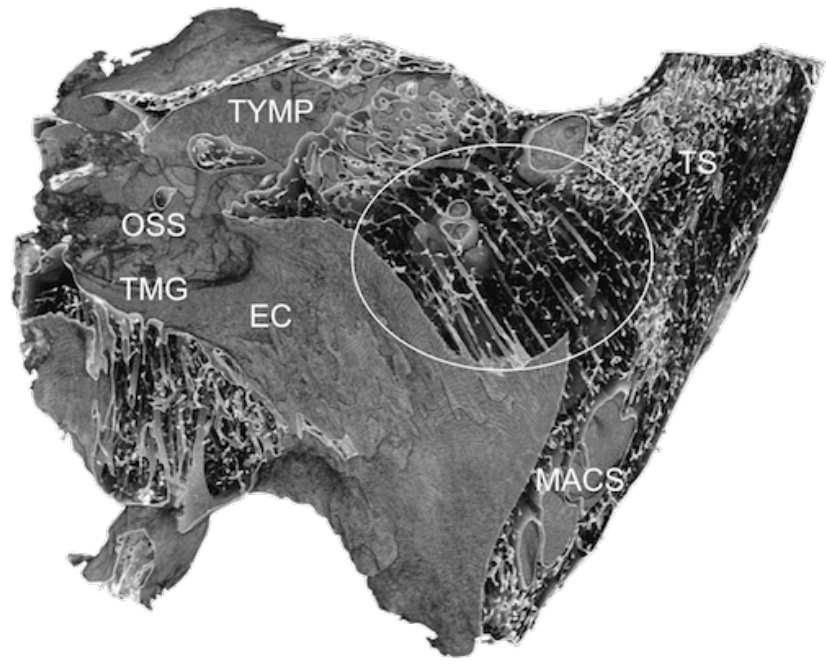

Fig. 2. Micro-channels observed between the surface of the temporal bone and its inner structures while modifying the transfer function of the volume rendering scheme[1]. Legend: (EC) ear canal, (MACS) mastoid air cell system, (OSS) ossicles, (TMG) tympanic membrane groove, (TS) trabecular spaces, (TYMP) tympanum.

\section{MATERIAL}

One human temporal bone specimen was used in this study. The bone specimen was scanned in Ghent at the Department of Physics and Astronomy, Belgium. The procedure used for scanning the temporal bone was similar to [4] and resumed in a previous study [1]. The micro-CT scan was cropped from an original size of $1820 \times 1820 \times 1211$ voxels down to a volume of $300 \times 300 \times 220$ voxels. The size of each voxel is $46 \mu \mathrm{m}$ isotropic. This test volume was chosen to represent microchannels in the proximity of mastoid air cells at the vicinity of the ear canal, with a large variety of sizes and shapes.

\section{METHODS}

Structures in a 3D volume can be represented using a structure tensor by exploiting its eigenvalues and eigenvectors, [5]. The tensor can be constructed as

$$
\mathbf{T}=\sum_{k=1}^{3} \lambda_{k} \hat{\mathbf{e}}_{k} \hat{\mathbf{e}}_{k}^{T} \text { with } \lambda_{k} \geq \lambda_{k+1} \geq 0 .
$$

Three specific cases of the structure tensor where $\lambda_{1} \geq$ $\lambda_{2} \geq \lambda_{3} \geq 0$ are the eigenvalues in decreasing order, $\hat{\mathbf{e}}_{i}$ the eigenvector corresponding to $\lambda_{i}$, and where $\mathbf{I}$ is the identity matrix $\mathbf{I}=\hat{\mathbf{e}}_{1} \hat{\mathbf{e}}_{1}^{T}+\hat{\mathbf{e}}_{2} \hat{\mathbf{e}}_{2}^{T}+\hat{\mathbf{e}}_{3} \hat{\mathbf{e}}_{3}^{T}$, can be extracted as:

- $\lambda_{1}>0, \quad \lambda_{2}=\lambda_{3}=0, \mathbf{T}_{1}=\lambda_{1} \hat{\mathbf{e}}_{1} \hat{\mathbf{e}}_{1}^{T}$. This case corresponds to a neighbourhood that is perfectly planar. The walls of the mastoid air cells are for instance represented through this rank 1 tensor.

- $\lambda_{1}=\lambda_{2}>0, \quad \lambda_{3}=0, \quad \mathbf{T}_{2}=\lambda_{1}\left(\mathbf{I}-\hat{\mathbf{e}}_{3} \hat{\mathbf{e}}_{3}^{T}\right)$. This case corresponds to a neighbourhood that is constant along lines and/or tubular structures. The micro-channels are represented through this rank 2 tensor.
- $\lambda_{1}=\lambda_{2}=\lambda_{3}>0, \quad \mathbf{T}_{3}=\lambda_{1} \mathbf{I}$. This case corresponds to an isotropic neighbourhood, meaning that there exists energy without any specific orientation in the neighbourhood, e.g. in the case of noise. This case is a rank 3 tensor.

The three cases are illustrated in Fig. 3. The probability of

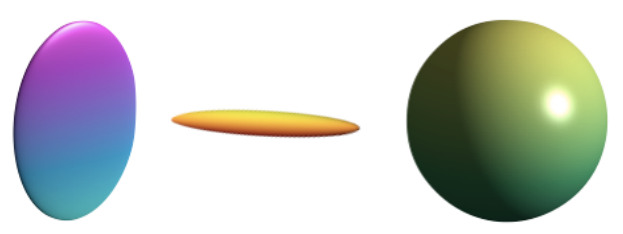

Fig. 3. The three cases: planar represented as a vertical disk (left) linear/tubular case represented as a cigar-like shape (middle), and isotropic case represented as a sphere (right).

each neighbourhood belonging to either a rank 1, rank 2, or a rank 3 tensor, as defined in [5] and recalled from [3], can further be investigated from the local structure tensor by $\mathrm{p} 1$, $\mathrm{p} 2$, and $\mathrm{p} 3$, where $\sum_{k=1}^{3} p_{k}=1$, as

$$
p_{1}=\frac{\lambda_{1}-\lambda_{2}}{\lambda_{1}}, p_{2}=\frac{\lambda_{2}-\lambda_{3}}{\lambda_{1}}, p_{3}=\frac{\lambda_{3}}{\lambda_{1}} .
$$

A combination of these three cases into an ideal structure tensor is given by

$$
\mathbf{T}=p_{1} \mathbf{T}_{1}+p_{2} \mathbf{T}_{2}+p_{3} \mathbf{T}_{3}
$$

Several examples of rank 1, rank 2, rank 3 tensors are given in Fig. 4 for several ellipsoids of different sizes and orientations. The rank 1 tensor is displayed as blue (planar), the rank 2 tensor is mapped with the red color (linear or tubular), and the rank 3 tensor is rendered green (isotropic). In Fig. 4(A), the original data is represented. The three cases rank 1 , rank 2 , and rank 3 are illustrated respectively in Fig. 4(B), Fig. 4(C), and Fig. 4(D). Fig. 4(E) and Fig. 4(F) demonstrate the combination of rank 1 and rank 2 tensors and the combination of rank 2 and rank 3 respectively. Fig. $4(\mathrm{H})$ is a combination of all three rank tensors together with the original data placed next to it for easier comparison (Fig. 4(G)). In Fig. 4(H), a zoom at the tip of the thinnest ellipsoid is illustrated in the yellow square to point out the presence of a rank 3 tensor shown as a green sphere. Also note, that the isotropic part in green from Fig. 4(D) lies inside the planar part in Fig. 4(B). As stated in [3], to obtain rank 1, rank 2 and rank 3 tensors, a set of quadrature filter responses were computed over the whole volume so as to estimate the structure tensor given in Eq. 3. The advantage of using a quadrature filter approach is its property of phaseindependence, producing a filter response equally strong for odd and even structures, representing a micro-channel either as a line or an edge locally depending both on the size of the micro-channel and on the scale being chosen for the study. The typical frequency function $F$ used for a quadrature filter is given by

$$
F_{k}(\hat{\mathbf{u}})=R(\rho) D_{k}(\hat{\mathbf{u}}) .
$$


A

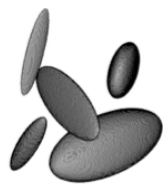

D



B

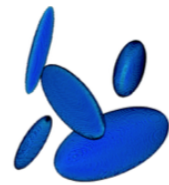

E

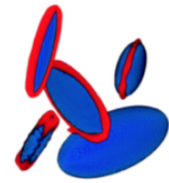

C

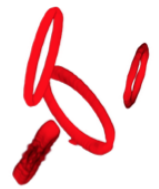

$\mathrm{F}$



G

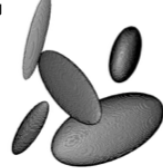

$\mathrm{H}$



Fig. 4. Results of structure tensor analysis on test objects to show the contribution of each case and their combination thereof. Legend: (A) Original data, (B) rank 1 tensor, (C) rank 2 tensor, (D) rank 3 tensor, (E) mixture of rank 1 and 2 tensors, (F) mixture of rank 2 and 3 tensors, (G) original data repeated for comparison with mixture of all rank tensors $(\mathrm{H})$. Observe the isotropic tiny sphere at the end tip of the smallest ellipsoid further zoomed in the yellow box.

The arbitrary but positive radial bandwidth function $R(\rho)$, typically a $\log$-normal function with $\rho=\|\hat{\mathbf{u}}\|$, together with a direction function $D_{k}(\hat{\mathbf{u}})$, allows the quadrature filter to be built as spherically separable in the Fourier domain to pick up energy in all possible orientations. An example of a lognormal $R(\rho)$ is illustrated in Fig. 5(A). To determine in what scale the filter has its sensitivity, the radial bandwidth function can be designed as a bandpass function with a certain centre frequency and bandwidth. The direction function varies as $\cos ^{2}(\phi), \phi$ being the angle between $\mathbf{u}$ and the filter direction $\mathbf{n}_{k}$, and is given by

$$
D_{k}(\mathbf{u})=\left\{\begin{array}{ll}
\left(\hat{\mathbf{u}} \cdot \hat{\mathbf{n}}_{k}\right)^{2} & \text { if }\left(\hat{\mathbf{u}} \cdot \hat{\mathbf{n}}_{k}\right) \geq 0 \\
0 & \text { otherwise }
\end{array},\right.
$$

where $\hat{\mathbf{n}}_{k}$ is the direction vector of each quadrature filter. The magnitude of the quadrature filter output $\left|q_{k}\right|$ can then obtained by computing

$$
\left|q_{k}\right|=\left\|\int S(\mathbf{u}) F_{k}(\mathbf{u}) d \mathbf{u}\right\|,
$$

where $S(\mathbf{u})$ is the Fourier transform of the original data. The structure tensor can now be estimated as

$$
\mathbf{T}_{e s t}=\sum_{k=1}^{6}\left|q_{k}\right|\left(\alpha \hat{\mathbf{n}}_{k} \hat{\mathbf{n}}_{k}^{T}-\beta \mathbf{I}\right),
$$

where $\left|q_{k}\right|$ is the magnitude of the filter output, and $\hat{\mathbf{n}}_{k}$ is the direction vector for the quadrature filter $k$ [5]. For $3 \mathrm{D}, \alpha=\frac{5}{4}$ and $\beta=\frac{1}{4}$. The magnitude of the structure tensor is illustrated in Fig. 5(B). When carefully observing the results from the previous study [3], the very thin microchannels are not fully represented along their path. The noise further corrupts their definition. A natural step is, therefore, to reduce the noise level as much as possible, while at the same time enhancing the weak micro-channels by favoring the linear/tubular structure information from the local structure tensor analysis. This process is known as anisotropic adaptive filtering and has been used previously in $2 \mathrm{D}, 3 \mathrm{D}$, and even 4D [7], [8], [9], [10]. Similar to [8], the enhanced signal $f_{a}$ is defined as

$$
f_{a}=f_{l p}+\sum_{k=1}^{6} w_{k} f_{k}
$$

where $f_{l p}$ is the lowpass filter response, $w_{k}$ are scalar weights and $f_{k}$ are the responses from the bandpass filters in different directions. To express the adaptive filter in terms of the local structure tensor, the scalar weights $w_{k}$ can be estimated by projection of the local structure tensor field onto a set of dual tensors $M_{k}$ corresponding to $\left(\alpha \hat{\mathbf{n}}_{k} \hat{\mathbf{n}}_{k}^{T}-\beta \mathbf{I}\right)$ in the right hand side of Eq. 7. The enhanced signal $f_{a}$ can then be written as

$$
f_{a}=f_{l p}+\sum_{k=1}^{6}\left\langle\tilde{\mathbf{T}}, \mathbf{M}_{k}\right\rangle f_{k},
$$

Smoothing the components of $\mathbf{T}_{\text {est }}$ using a small isotropic low-pass kernel prevents rapid changes in the tensor field. A soft threshold of the norm of the low-pass filtered tensor is applied according to

$$
\tilde{\mathbf{T}}=\frac{\gamma\left(\left\|\mathbf{T}_{l p}\right\|\right)}{\left\|\mathbf{T}_{l p}\right\|} \mathbf{T}_{l p}
$$

where $\mathbf{T}_{l p}=h_{l p} * \mathbf{T}_{e s t}$. The function $\gamma$ is a smooth mapping corresponding to low-parametric sigmoid function defined for $\alpha \in[0,1]$ as

$$
\gamma(x, \sigma ; \alpha, \beta)=\frac{x^{\beta}}{x^{\alpha+\beta}+\sigma^{\beta}} .
$$

The $\gamma$-function controls the amount of anisotropic high-pass filtering, allowing a bandwidth selectivity. Well defined structures are therefore represented by a large norm while small fluctuations, such as noise, are represented by a small norm. $\alpha$, typically in a range of $[0,0.5]$, is used to accentuate weak structures that are slightly above the noise level threshold $\sigma$. $\beta$ sets the slope of the $\gamma$ function in the noise-signal transition [5]. The output of the $\gamma$-function corresponds to the largest eigenvalue, i.e. $\gamma_{1}$, of the control tensor $\mathbf{C}$. To control the shape of the adaptive filter, i.e. the orientational variation in the high-pass regions, the ratio of the eigenvalues of the local structure tensor can be used and remapped using the $\mu$-function as

$$
\mu(x ; \alpha, \beta)=\left[\frac{(x(1-\alpha))^{\beta}}{(x(1-\alpha))^{\beta}+(\alpha(1-x))^{\beta}}\right],
$$

where $x=\frac{\lambda_{n}}{\lambda_{n-1}}$. $\alpha$ is defined in the $\mu$-function as the value of $\frac{\lambda_{n}}{\lambda_{n-1}}$ for which the $\mu$-function is $\frac{1}{2}$. $\beta$ defines the slope in the transition area in the same manner as for the $\gamma$-function in Eq. 11. The resulting $\mu_{n}$ will correspond to the filter shape coefficients. A control tensor can now be constructed based on the largest eigenvalue $\gamma_{1}$ and the filter shape coefficients. Reusing the eigensystem from the original local structure tensor ensures a preservation of the orientation estimation. 
Therefore, the control tensor can be written in the $3 \mathrm{D}$ case as

$$
\mathbf{C}=\gamma_{1}\left[\hat{\mathbf{e}}_{1} \hat{\mathbf{e}}_{1}^{T}+\mu_{2}\left[\hat{\mathbf{e}}_{2} \hat{\mathbf{e}}_{2}^{T}+\mu_{3}\left[\hat{\mathbf{e}}_{3} \hat{\mathbf{e}}_{3}^{T}\right]\right]\right],
$$

where $\hat{\mathbf{e}}_{n}^{T}$ are the eigenvectors of $\mathbf{T}_{l p}$. Eq. 13 can then be rewritten as a weighted sum of outer products of $\mathbf{T}_{l p}$ as

$$
\mathbf{C}=\sum_{n=1}^{3} \gamma_{k} \hat{\mathbf{e}}_{n} \hat{\mathbf{e}}_{n}^{T},
$$

leading to a modification of Eq. 9 such as

$$
f_{a}=f_{l p}+\sum_{k=1}^{6}\left\langle\mathbf{C}, \mathbf{M}_{k}\right\rangle f_{k} .
$$

An example of the magnitude of the control tensor is given in Fig. 5(C). A high-pass amplification factor was further added to Eq. 15 to favour enhancement of solely linear or tubular structures by using the eigenvalues from the structure tensor according to

$$
f_{a}=f_{l p}+a_{h p} \sum_{k=1}^{6}\left\langle\mathbf{C}, \mathbf{M}_{k}\right\rangle f_{k},
$$

where $a_{h p}$ is defined as

$$
a_{h p}=\zeta \times\left(\frac{2 \lambda_{1}+\lambda_{2}-\lambda_{3}}{3 \lambda_{1}-\lambda_{2}+\lambda_{3}}\right),
$$

with $\zeta=1.3$ in this study. The enhanced data can be seen as the sum of a low-pass filtered version of the original data, applied only once, with a linear combination of anisotropic high-pass filters using the corresponding orientations from the quadrature filter, and where the scalar weights $w_{k}$ are calculated using the control tensor.
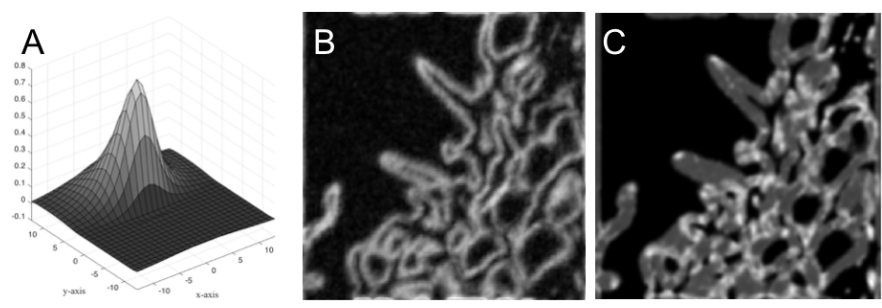

Fig. 5. Intermediate results. (A) Log-normal function for the quadrature filter in one of the six directions (Eq. 4). (B) Magnitude of the structure tensor estimated from the original data (Eq. 7). (C) Magnitude of the resulting control tensor (Eq. 13). For reference, the original data is given in Fig. 6.

\section{RESUlTS \& DiscusSiON}

Visual comparison of the enhanced data versus the original data is first provided in 2D as shown in Fig. 6. To compare with already existing methods, a free Matlab implementation of an anisotropic diffusion PDE scheme was used with the following parameters: Scheme : $c E E D, \lambda_{\text {weickert }}=0.0025$, Time $_{\text {final }}=0.5$, while the remaining options were kept by default. Further details about this scheme are given in [13]. This anisotropic diffusion scheme was found to be the closest method to compare to in relation to our algorithm, since it relies on stencils with 6-points locally adapted to a diffusion tensor.

In the first column of Fig. 6, the original data is shown with the second row being a zoomed part of the upper corner of the top row. In the second column, the results from the current study are illustrated. In the third column, results from using the anisotropic diffusion are provided. Results from both studies look very similar. Both methods show a reduction of the noise level while preserving the micro-channels. Fig. 7

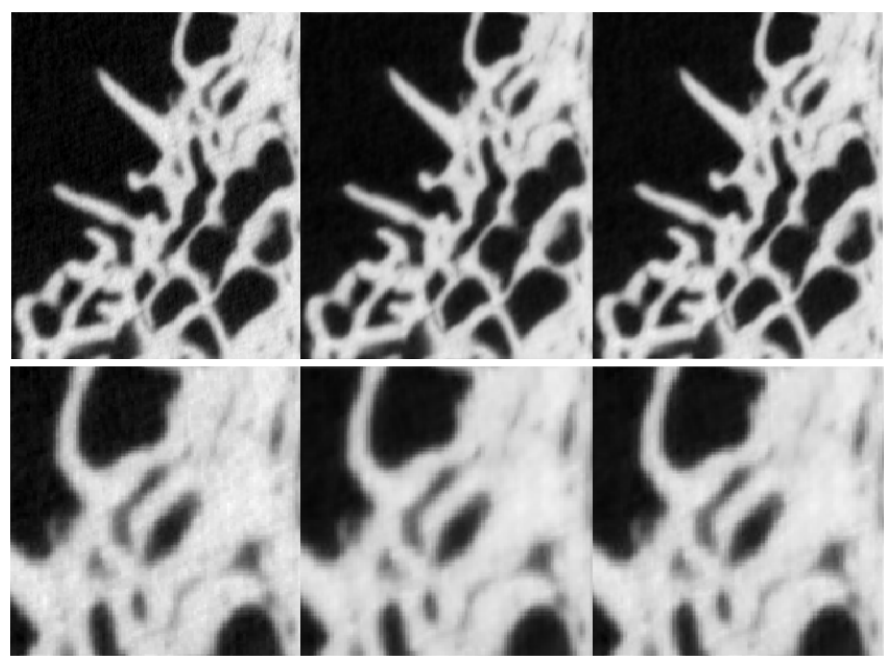

Fig. 6. Comparison of the adaptive filtering used in this study (middle column) with an anisotropic diffusion scheme from [13] (right column) with a slice from the original data (left column). The second row illustrates a zoomed part of the partial 2D slice from the upper row at the upper right corner.

gives a $3 \mathrm{D}$ representation of the mixture of rank 1 , rank 2 , and rank 3 tensors. Fig. 7 is a mapping of the three rank tensors based on the lower intensities, i.e. air and soft tissues, from the respective data using a soft mask. This masking makes it possible to know what type of rank tensor, and their subsequent mixtures, the tubular structures, i.e. the microchannels, are made of. In Fig. 7, the first row illustrates the mixture of the three rank tensors computed from $\mathbf{T}_{\text {est }}$ (Eq. 7) from the original data. Similarly, in the second row, the mixture of $p_{1}, p_{2}, p_{3}$ rank tensors from the enhanced data is shown. In the third row, the same is applied for the enhancement via the anisotropic diffusion PDE scheme. To appreciate more details from the resulting $p_{1}, p_{2}, p_{3}$ rank tensors mixture, the right column gives a zoomed version of the left column based on the dashed squared at the lower right corner on each row. The top row is used as a reference. Although the results from the current study are very similar to the results from the anisotropic diffusion scheme, it can be noticed that the tubular structures appear more complete especially when compared to $p_{1}, p_{2}, p_{3}$ rank tensors mixture from the previous study [3].

A visual inspection of the enhancement on the bone is illustrated in Fig. 8. The original data is shown in the first row; the enhancement achieved in the current study is shown in the second row, while the enhancement from the anisotropic 

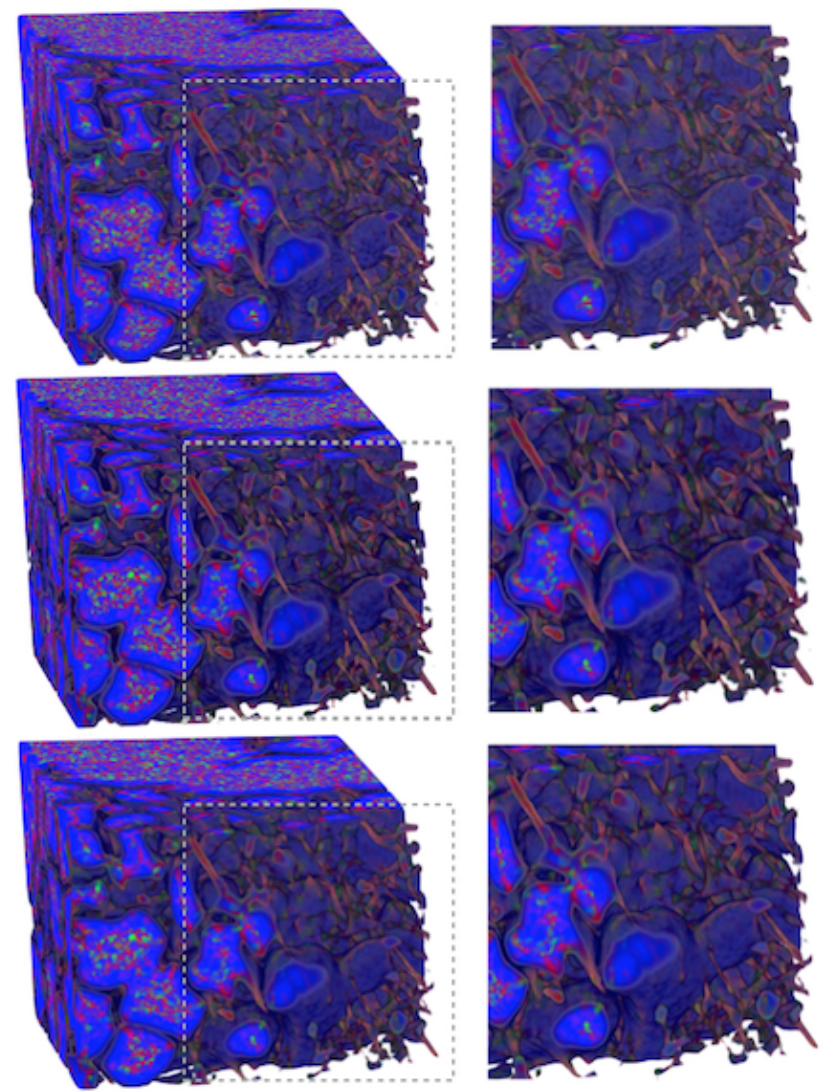

Fig. 7. Soft masking of the $p_{1}, p_{2}, p_{3}$ rank tensors only applied on the low gray values from the respective data so as to show the content of the micro-channels and the transition to the neighboring air cells. The first row corresponds to the rank 1 , rank 2, and rank 3 tensors from the original data. The second row corresponds with the enhanced using the current scheme while the third row corresponds to the anisotropic diffusion PDE based scheme. The right column is a further zoom from the dashed square shown in the left column at the lower right corner of the $3 \mathrm{D}$ volume.

diffusion scheme is represented in the third row. A zoomed version of the lower right corner is also available to appreciate how well the structures are preserved while the noise is reduced. Using a common transfer function, it is almost impossible to see any difference between the two enhancement schemes. The results from the current study appear with a slightly higher contrast for the weak structures though.

Using a different setting for the transfer function, similar to [3], visualization of the micro-channels from the enhanced data can be visually compared to the original data (top row), see Fig. 9. Removal of the noise combined with enhancement of the tubular structures, i.e. micro-channels, make them appear more structured and to have a more coherent connectivity. The enhanced data resulting from the current study is represented in the second row and the enhancement emanating from the anisotropic diffusion scheme is illustrated in the bottom row. A zoom of the lower right corner is also available to better assess the variation between the results, both comparable with the original data.

Although relevant, a quantitative analysis was not performed
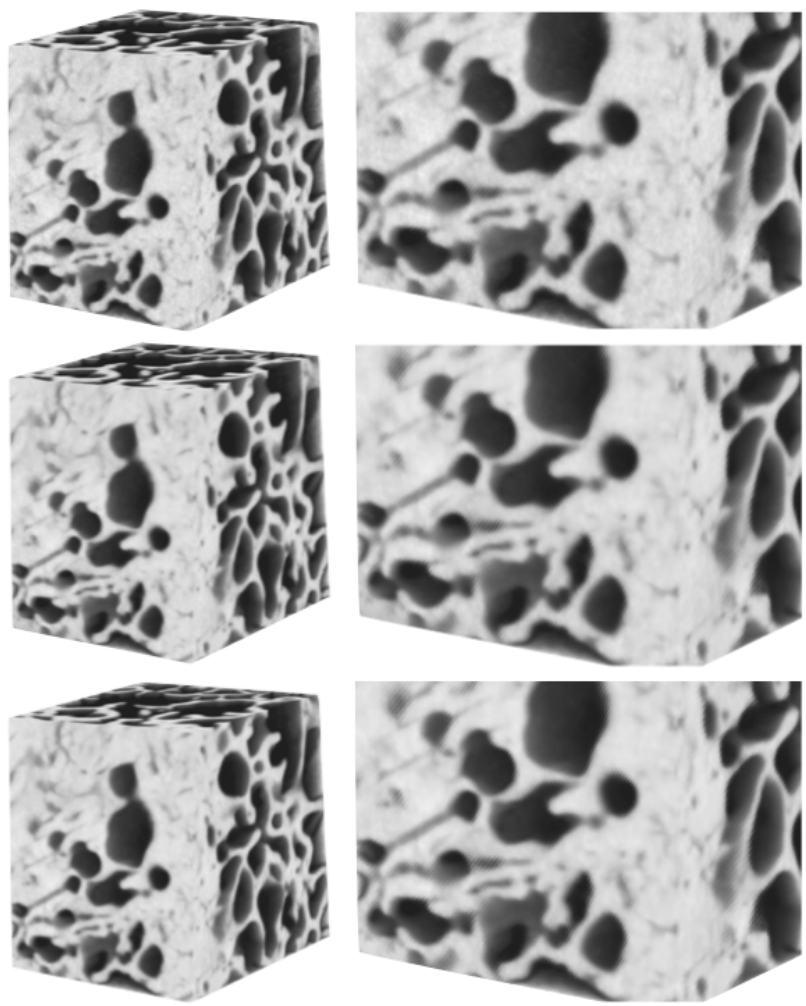

Fig. 8. Comparison of the enhancement used in the current study (middle row) with the original data (top row) along with the anisotropic diffusion scheme from [13] (bottom row). The right column gives a further zoom of the lower right corner of the $3 \mathrm{D}$ volume.

simply due to the high complexity of the data. To obtain relevant statistics from a quantitative analysis from such a study, the bone specimens should be scanned several times with the same settings and processed with the same scheme for the adaptive enhancement to be able to measure how much variation there is in the results. Although the bone specimens were fixated, they will shrink during the overall experiment.

A comparison with anisotropic diffusion filtering was performed. Despite the fact that the present scheme requires adjustments of many parameters, the processing time for a given set of quadrature filters was considerably faster than the anisotropic diffusion scheme, due to the iterative feature of the diffusion process. For the current data size, the anisotropic diffusion scheme was three times slower than the current scheme. An ITK version of the anisotropic diffusion scheme exists but the Matlab version was used for easier comparison purposes.

Besides the fact that our proposed algorithm has many parameters to adjust as compared to the anisotropic diffusion scheme, a better control on how to perform the enhancement is possible. The adaptive filtering is believed to be better for the tubular structures, compared to the anisotropic diffusion. Metrics such as Structural Similarity Index (SSIM), Quality Index based on Local Variance (QILV) or the Mean Squared Error (MSE) can be used such as in [12], [11] and were investigated in the current study, but because the varying size 

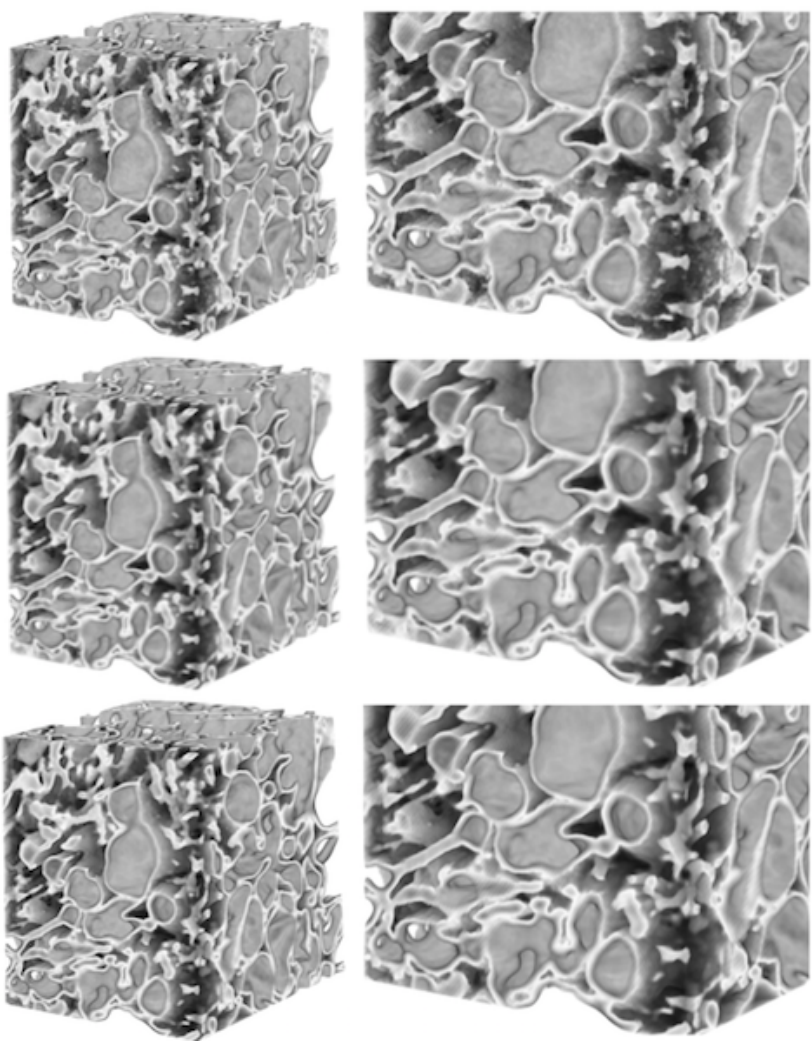

Fig. 9. Comparison of the enhancement used in the current study (middle row) with the original data (top row) along with the anisotropic diffusion scheme from [13] (bottom row) similar to Fig. 8 but with a transfer function defined in [3]. The right column gives a further zoom of the lower right corner of the $3 \mathrm{D}$ volume.

of the structures to enhance can sometimes be close to the noise level there is a need to preserve a certain amount of noise in the spectrum leading to inconclusive results in the present work.

Investigation of the residuals between the original data and the enhanced data using both methods was also performed. Combination of both noise reduction and enhancement of the weak tubular structures using the current scheme does not permit to distinguish the contribution of either part of the scheme. The residual was first computed for the sole adaptive filtering without enhancement of the tubular structure, see Fig. 10(A). The residual for the complete scheme was computed as illustrated in Fig. 10(B). Finally, the residual from the anisotropic diffusion scheme was estimated, see Fig. $10(\mathrm{C})$. Interestingly, the residual from the anisotropic diffusion scheme slightly shows the silhouette of the bone as in the case of Fig. 10(B) induced by the enhancement part. It is more challenging to observe any structure when only the adaptive filtering was used in the current scheme.

\section{CONCLUSiON}

This study has demonstrated the positive effect of enhancing the micro-channels while reducing the noise level in the original data using an adaptive filtering scheme together with


Fig. 10. Residuals. (A) Residual estimated only using the adaptive filtering (B) Residual using both the adaptive filtering together with enhancement. (C) Residual from the anisotropic diffusion scheme from [13].

a structure tensor analysis. Reduction of the noise enabled better observation of the transition between the MACS and the micro-channels. The enhancement, further helped by amplification of tubular structures, allowed some micro-channels to recover their full paths.

\section{ACKNOWLEDGMENT}

This research has been supported by The Obel Family Foundation (Denmark).

\section{REFERENCES}

[1] O. Cros, M. Borga, E. Pauwels, J.J.J. Dirckx and M. Gaihede. Microchannels in the mastoid anatomy. Indications of a separate blood supply of the air cell system mucosa by micro-CT scanning. Hearing Research, 301: 60-65, 2013.

[2] M. Gaihede, O. Cros and S. Padurariu. The role of the mastoid in middle ear pressure regulation. In H. Takahashi (Ed.), Cholesteatoma and Ear Surgery - An Update: Proceedings of the 9th International Conference on Cholesteatoma and ear Surgery, proceeding, 17-20, Kugler Publications, 2013.

[3] O. Cros, M. Gaihede, M. Andersson and H. Knutsson. Structual Analysis of Micro-channels in Human Temporal Bone. In IEEE 12th International Symposium on Biomedical Imaging (ISBI), proceedings, New York, April 2015.

[4] B.C. Masschaele, V. Cnudde, M. Dierick, P. Jacobs, L. Van Hoorebeke and J. Vlassenbroeck. UGCT: new X-ray radiography and tomography facility. Nuclear Instruments and Methods in Physics Research, 580(A) : 266-269, 2007.

[5] G. Granlund and H. Knutsson. Signal processing for computer vision. Kluwer Academic Publishers, Dordrecht, ISBN: 0792395301, 1995.

[6] H. Knutsson and R. Wilson and G. Granlund. Anisotropic non-stationary image estimation and its applications: Part I-Restoration of noisy images. IEEE Transactions on Communications, 31(3) : 388-397, 1983.

[7] L. Haglund. Adaptive Multidimensional Filtering. PhD thesis, Linköping Studies in Science and Technology, Dissertations No. 284, Linköping, 1992.

[8] B. Svensson. A Multidimensional Filtering Framework with Applications to Local Structure Analysis and Image Enhancement. $\mathrm{PhD}$ thesis, Linköping Studies in Science and Technology, Dissertations No. 1171, Linköping, 2008.

[9] A. Maier, L. Wigstrom, H.G. Hofmann, J. Hornegger, L. Zhu, N Strobel and R. Fahrig. Three-dimensional anisotropic adaptive filtering of projection data for noise reduction in cone beam CT. Medical Physics, 38(11) : 5896-5909, 2011.

[10] A. Eklund, M. Andersson and H. Knutsson. True 4D Image Denoising on the GPU. International Journal of Biomedical Imaging, 2011 : 1-16, 2011.

[11] Z. Hossain and T. Möller. Edge Aware Anisotropic Diffusion for 3D Scalar Data. IEEE Transactions On Visualization and Computer Graphics, 16(6) : 1376-1385, 2010.

[12] Z. Wang, A.C. Bovik, H.R. Sheikh and E.P. Simoncelli. Image Quality Assessment: From Error Visibility to Structural Similarity. IEEE Transactions on Image Processing, 13(4) : 1-14, 2004.

[13] J. Fehrenbach and J-M Mirebeau Sparse Non-negative Stencils for Anisotropic Diffusion. Journal of Mathematical Imaging and Vision, 49(1), 123-147, 2014. 